\title{
Role of drug metabolism \& disposition in discovery and development of new drugs
}

\author{
Nirmal Joshi ${ }^{1}$, Rajeshwar Kamal Kant Arya ${ }^{2}$, Dheeraj Bisht ${ }^{2}$, Deepak Chandra Joshi ${ }^{3}$ \\ ${ }^{1}$ Amrapali Institute of Pharmacy and Sciences, Shiksha Nagar, Lamachaur, Haldwani, \\ Nainital, India \\ ${ }^{2}$ Department of Pharmaceutical Sciences, Kumaun University Campus Bhimtal, Nainital, \\ India \\ ${ }^{3}$ Department of Pharmacy, Invertis University, Bareilly, UP, India
}

Received: 12-10-2021 / Revised Accepted: 28-11-2021 / Published: 01-12-2021

\begin{abstract}
Drug development is an intrinsically risky business. Like a high stakes poker game the entry costs are high and the probability of winning is low. Indeed, only a tiny percentage of lead compounds ever reach USFDA approval. At any point during the drug development process a prospective drug lead may be terminated owing to lack of efficacy, adverse effects, excessive toxicity, poor absorption or poor clearance. Unfortunately, the more promising a drug lead appears to be, the more costly it is to terminate its development. Typically, the cost of killing a drug grows exponentially as a drug lead moves further down the development pipeline. As a result there is considerable interest in developing either experimental or computational methods that can identify potentially problematic drug leads at the earliest stages in their development. One promising route is through the prediction or modeling of ADME (absorption, distribution, metabolism and excretion). ADME data, whether experimentally measured or computationally predicted, provide key insights into how a drug will ultimately be treated or accepted by the body. So while a drug lead may exhibit phenomenal efficacy in vitro, poor ADME results will almost invariably terminate its development. This review focuses on the use of ADME modeling to reduce late-stage attrition in drug discovery programmes. It also highlights what tools exist today for visualising and predicting ADME data, what tools need to be developed, and the importance of integrating ADME data to aid in compound selection during the earliest phases of drug discovery. It also discusses what kinds of tools need to be developed, and the importance of integrating ADME data to aid in compound selection during the earliest phases of drug discovery.
\end{abstract}

Keywords: Drug development, USFDA, ADME, Drug Discovery

Address for Correspondence: Nirmal Joshi, Amrapali Institute of Pharmacy and Sciences, Shiksha Nagar, Lamachaur, Haldwani, Nainital, India

How to Cite this Article: Nirmal Joshi, Rajeshwar Kamal Kant Arya, Dheeraj Bisht, Deepak Chandra Joshi. Role of drug metabolism \& disposition in discovery and development of new drugs. World J Pharm Sci 2021; 9(12): 180-190; https://doi.org/10.54037/WJPS.2021.91207

Copyright:2021@ The Author(s). This is an open access article distributed under the terms of the Creative Commons AttributionNonCommercial-ShareAlike 4.0 International License (CC BY-NC-SA), which allows re-users to distribute, remix, adapt, and build upon the material in any medium or format for noncommercial purposes only, and only so long as attribution is given to the creator. If you remix, adapt, or build upon the material, you must license the modified material under identical terms. 


\section{INTRODUCTION}

Drug Discovery Process: The drug discovery process begins with first choosing a specific disease to treat. Understanding something about the biochemical pathway(s) or genetics of the disease allows researchers to focus on a specific class of enzymes or receptors potentially responsible for the disorder. After identifying and validating the target enzyme/receptor, a high-throughput screen of potentially millions of small molecules is conducted. The results of this screening allow medicinal chemists to focus only on those molecules containing certain functional groups, core structures and 3D shapes. These leads are then subjected to further biochemical and in vitro assays to eliminate molecules with potential side effects, drug-drug interactions or toxicity. A select few candidates are pursued further during the lead optimization phase. Herein, medicinal chemists fine tune the reactivity and structure of these ligands. These leads are again tested using both in vitro and in vivo methods. This cycle of chemical fine tuning and biological testing is repeated several times until the most likely molecule to succeed in clinical trials has been synthesized and characterized as best as possible. The optimized molecule is then chosen to be subjected to more extensive studies for IND filing; meanwhile, the scale-up process synthesis is executed. This assessment involves bioanalytical quantification of metabolites, plasma concentration measurements, regular vet assessments, and ultimately necropsy of several organs. These more extensive in vivo ADME studies are then used to estimate the PK/PD parameters in humans and also provide guidance for physicians in clinical trials. A schematic flowchart of this process is shown in Figure 1.

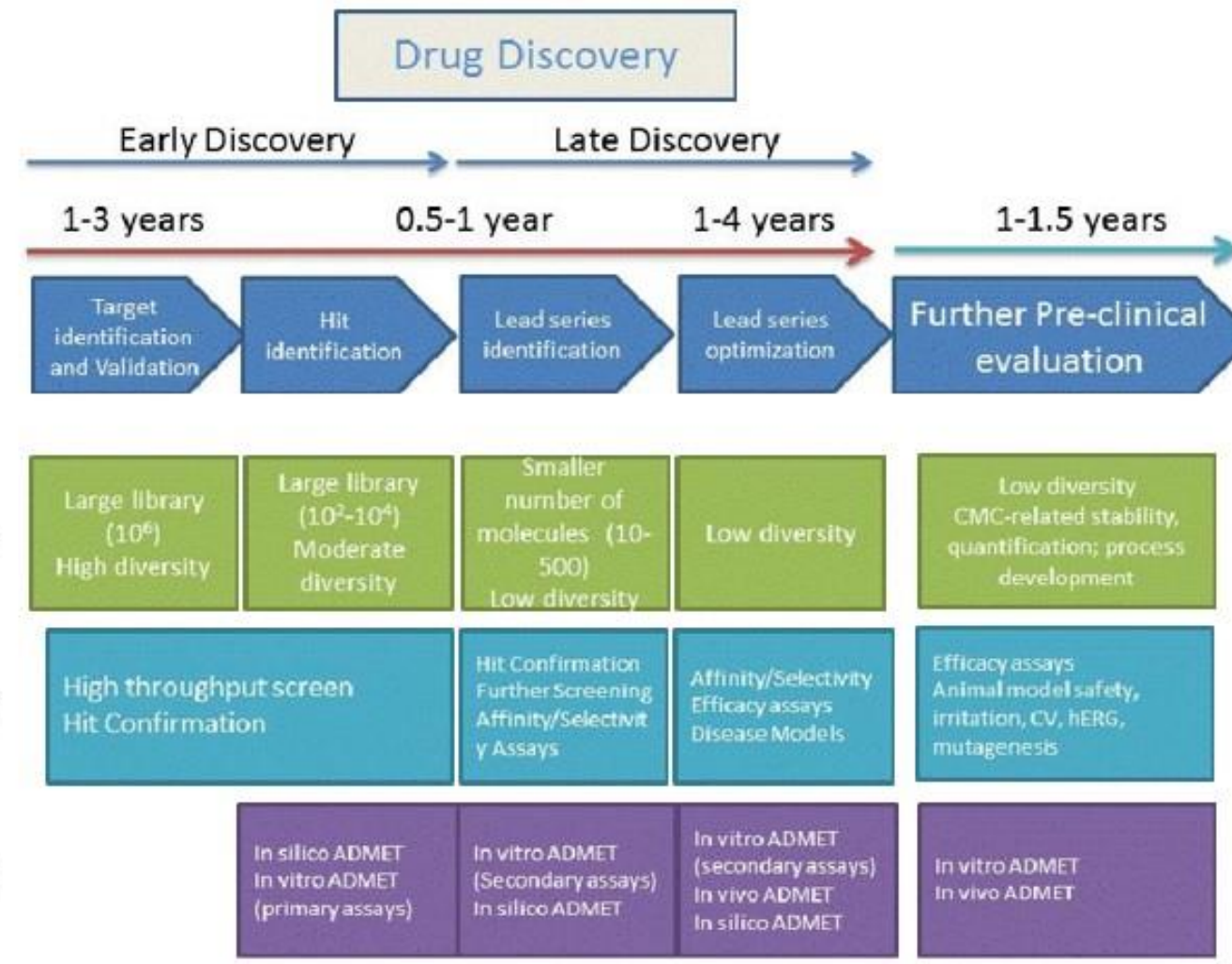

Figure1. Compounds in a chemical library are screened to identify those molecules that interact with the intended target. Molecules that are positive in this assay as "Hits" begin the process of lead identification (Hitto-Lead) and Lead optimization followed by further preclinical tests. Along the way different ADME/T assays are carried out with varying complexity.

\section{Drug Failure: Fail Early, Fail Cheap}

The discovery and development of a new pharmaceutical drug requires more than a billion dollars and can take 12 years of research effort (Adams and Brantner 2010; DiMasi, Hansen, and Grabowski 2003). The drug discovery and development process can be divided into four distinct phases: early discovery, late discovery, preclinical, (Fig.1) and finally the clinical trials.
Although there are several factors that contribute to attrition, two major reasons are efficacy and toxicity. It has been estimated that only 1 of 10,000 chemicals that enter the discovery process ever reaches the market. This is not hard to imagine when one considers that $40 \%$ of NCEs that begin preclinical safety studies in animals will fail due to toxicity, and that $89 \%$ of NCEs that enter clinical trials will fail. The greatest cost in terms of time 
occurs in early and late discovery, which can require 6 to 8 years of research effort. In comparison, the highest cost in terms of dollars occurs in the preclinical and clinical studies (DiMasi, Hansen, and Grabowski 2003; Kletter et al. 2013; McCarter et al. 2013). The ability to identify and reduce risk early can significantly improve the process of drug development by improving efficiency and improving the probability of success. The new paradigm in drug discovery should include a robust means of identifying issues related to toxicity early in the discovery process where the cost of dropping a molecule is less than in later phases.

\section{Preclinical ADME/T Testing:}

As discussed above, every potential drug molecule needs to be tested for ADME/T properties. In this section, we briefly describe the commonly performed in vitro and in vivo ADME/T studies.

\section{In vitro Assays}

ADME/T studies are done throughout the drug discovery and development process (Guttendorf). They can be done in preclinical studies leading up to an IND or be assessed in humans during clinical trials to file an NDA (Guttendorf). In vitro ADME studies are typically conducted at the same stage as in vivo $\mathrm{PK}$ studies to facilitate selection of drug candidates with the best safety and pharmacological profile while understanding the mechanisms behind their activity (Steinmetz and Spack 2009).

\section{A. Absorption \& Distribution:}

Most orally bioavailable drugs are absorbed via the intestine. To understand pathways behind intestinal permeability ( $\mathrm{Li} \mathrm{2005)}$, the following assays are usually conducted:

Artificial membranes: These are artificial lipid bilayers used to measure the absorption of drugs across lipid membranes by passive diffusion. One example of such a membrane is PAMPA (Parallel Membrane Permeability Assay) which can be done on a high throughput basis. The drug is filtered through a hydrophilic membrane in the donor chamber into the recipient chamber. This ability to permeate is measured by UV analysis of the drug present in the recipient chamber (Li 2005).

In vitro cell culture assays: As PAMPA measures only passive diffusion and does not consider active uptake and transport, cell culture systems which can mimic in vivo intestinal epithelium are required to provide insights into mechanisms involved in drug absorption ('Note for Guidance on
Toxicokinetics'). These assays can be performed using a similar technique as PAMPA, by culturing on a plate ('Preclinical Safety Evaluation of Biotechnology- Derived Pharmaceuticals'). The following cell culture systems are used:

Caco-2: Human colon carcinoma cells are cultured to measure active efflux ('Preclinical Safety Evaluation of Biotechnology-Derived Pharmaceuticals') of drugs that are pumped back into the lumen after being absorbed in the intestine (Li 2005). An important transporter involved in this mechanism is P-gp(Li 2005), which is assayed by measuring active efflux of drugs like verapamil or cylcosporin('Preclinical Safety Evaluation of Biotechnology-Derived Pharmaceuticals')

MDCK (Madine Darby Canine Kidney): This cell line is also used to measure drug efflux ('Testing of Carcinogenecity of Pharmaceuticals'). They are used in conjunction with Caco- 2 because they can become confluent quickly ('Testing of Carcinogenecity of Pharmaceuticals'). This assay can be performed by either using wild- type MDCK cells or cells which have been transfected to express the MDR1 gene which expresses the Pgp transporter ('Testing of Carcinogenecity of Pharmaceuticals').The MDR1-MDCK cell line is used so that the involvement of only the P-gp transporter can be measured as compared to Caco-2 which expresses different transporters as it is derived from human intestinal epithelium ('Testing of Carcinogenecity of Pharmaceuticals'). Both wild type MDCK and Mdr1-MDCK assays are compared to eliminate any canine transporter activity.

Other transporters: Apart from P-gp, transporters such as organic anion transporter (OAT), organic anion transporting polypeptide (OATP), organic cation transporter (OCT), multi-drug resistance proteins (MDRP) and breast cancer resistant protein (BCRP) have been recommended to be studied by the FDA ('Need for Carcinogenecity Studies of Pharmaceuticals').

\section{B. Metabolism:}

It is important to understand how the body will metabolize a drug, and how quickly those changes will occur. Metabolites of a drug can be toxic, can have new efficacy properties, and/or can interact alter the metabolism of other co-administered drugs. The adverse effects of a drug may arise from its breakdown into toxic metabolites or can be associated to its interactions with other drugs already being administered to the patient ( $\mathrm{Li} 2005)$. To assess its safety profile and pharmacokinetics, in vitro metabolism studies are required ( $\mathrm{Li} 2005$ ). Drug metabolism is facilitated by phase I oxidation by cytochrome P450 mono-oxygenases and phase 
II conjugation by UDP-dependent glucoronosyl transferase (UGT) and phenol sulfotransferase (PST) (Li 2005).

To study drug metabolism, in vitro systems are constructed using tissue, cells and enzymes from human liver (Li 2005). Inter- species differences can also be determined by using other animal sources like rats, mice, guinea pigs or rabbits.

Liver microsomes: These sub- cellular fractions consisting of endoplasmic reticulum of the liver are utilized to study the activity of phase I enzymes ( $\mathrm{Li}$ 2005).

Hepatocytes: Primary hepatocytes from rat, dog, monkey and human have been used extensively to evaluate chemical and drug toxicity, metabolism, bioactivation, transporter interaction, intrinsic clearance, and other biochemical processes $(\mathrm{Li}, \mathrm{Qu}$, and May 2001; Brown et al. 2007; Hewitt et al. 2007; Wilkening, Stahl, and Bader 2003; Naritomi et al. 2003). An important advantage of primary hepatocytes is that NCEs can be evaluated in vitro with cells prepared from normal tissue. Because primary hepatocytes have both phase I and phase II drug metabolizing systems they have been used extensively to study drug metabolism, and toxicity (McKim 2010), (Li 2005). Primary hepatocytes can be used to identify compounds that undergo metabolic activation and to determine how these metabolites affect cellular toxicity (Maggs et al. 2004; Kaminski and Stevens 1992). Optimal growth of hepatocytes is usually achieved with culture plates that have been coated with extracellular matrix proteins, such as matrigel or collagen. Culture time is limited in most laboratories to a maximum of $72 \mathrm{~h}$ without significant loss of metabolic activity. Cytochrome P450 activity is typically inducible and present at constitutive levels with good metabolic capacity, provided the cells are of high quality and are cultured under conditions that optimize cell viability and maintain CYP activities.

The following assays are performed:

* Metabolic stability (Li 2005): This assay can be performed on microsomes, supernatant fraction containing microsomes and cytosol (S9) (Dash et al.) and hepatocytes ( $\mathrm{Li} 2005$ ). Since a drug has to stay in the body for a long period of time, its stability can be measured by its rate of clearance (Li 2005).

* Microsomal stability (Prestwich): Large numbers of microsomes pooled from various donors can be screened to measure clearance involving phase I and some phase II enzymes in a high throughput fashion (Prestwich).
* S9 stability (Dash et al.):S9 fractions contain both phase I and phase II enzymes as they are supernatants obtained during the process of extraction of microsomes, consisting of both microsomes and cytosol (Dash et al.). They are assayed by methods similar to microsomal stability assays (Dash et al.).

* Hepatocyte stability (Gomez-Lechon et al.): Hepatocytes are used as a secondary screen to measure intrinsic clearance of compounds which performed well in the primary screens on microsomes and S9 fractions (GomezLechon et al.). Cells from at least 3 donors are used in each well to reduce variability and are screened for both phase I and phase II enzymes (Gomez-Lechon et al.). Both fresh and cryopreserved hepatocytes can be used (Gomez-Lechon et al.).

Metabolite identification and profiling (Knop et al.): When a drug is incubated with microsomes, hepatocytes or plasma samples, metabolites formed in vitro can be identified using Liquid Chromatography/Mass Spectrometry (LC/MS)(Knop et al.), (Li 2005). Early identification of these metabolites is important to detect any harmful forms which can lengthen or halt the drug development process altogether (Bissell et al.). If such metabolites are found, then the structure of the compound can be modified to prevent their creation. Alternatively, favorable metabolites can lead to selection of the best in vivo animal model for further downstream studies ( $\mathrm{Li}$ 2005).

\section{Drug-drug Interactions:}

The administration of one drug can significantly alter the plasma concentration, half-life, or toxicity of another drug. This phenomenon is called a drugdrug interaction or DDI. Drugs that inhibit cytochrome P450 (CYP) enzymes, such as CYP3A4, or that can induce the production of CYP enzymes have a high probability of producing a DDI (Wienkers and Heath 2005; Ito, Brown, and Houston 2004; Ito et al. 2005). In the case of permeability, bioavailability, metabolic stability, and potential DDIs, it makes sense to build in vitro screening models focused on predicting human outcomes. The Food and Drug Administration (FDA) requires the submission of an Investigational New Drug (IND) package prior to manufacturing, transport across state lines, and testing in humans. A large part of the IND submission consists of animal safety studies. Typically, these studies are performed in a rodent (rat) and non-rodent species (dog) (Kola and Landis 2004).

According to FDA's guidance document on in vitro testing, "in vitro studies can frequently serve as an 
adequate screening mechanism to rule out the importance of a metabolic pathway and drug-drug interactions that occur via this pathway so that subsequent in vivo testing is unnecessary" (Liu, LeCluyse, et al.). A drug might increase or decrease the metabolic activity of a coadministered drug by inhibiting the metabolic enzymes involved ( $\mathrm{Li} 2005$ ). The most commonly studied enzyme isoforms are CYPs 1A2, 2C8,2C6, 2C9, 2C19,2D6,2E1 and 3A4 (Li 2005). The phase II enzyme UGT1A1 is also studied as it can be inhibited by drugs that are cleared by glucoronidation (Liu, Chism, et al.). Drug-drug interactions (DDIs) are evaluated by performing the following assays:

- Enzyme inhibition: Inhibition of Cytochrome P450 enzymes can be analyzed by incubating the drug in question with liver microsomes (Li 2005). Since liver microsomes express the whole panel of CyP450 enzymes as in vivo, they are ideal for this assay (Marion, Leslie, and Brouwer). A decrease in the metabolites produced at different concentrations of the drug can be monitored to calculate an $\mathrm{IC}_{50}$ value (Marion, Leslie, and Brouwer). The $\mathrm{K}_{\mathrm{i}}$ can also be measured for a specific enzyme to further determine the type of inhibition and any further potential in vivo effects (Marion, Leslie, and Brouwer). If the $\mathrm{IC}_{50}$ or $\mathrm{K}_{\mathrm{i}}$ values are low, that can indicate the drug can lead to DDIs (Li 2005).

- Enzyme induction: In this assay, primary hepatocytes are incubated with the drug being evaluated and CYP450 enzyme activity, protein or mRNA levels are measured ( $\mathrm{Li}$ 2005). If enzyme induction is significantly greater than that of controls, then the drug can lead to potential DDIs with other drugs (Mathijs et al.). Primary hepatocytes are used because mRNA level is also monitored to provide insights into the mechanism behind induction (Li 2005).

\section{Excretion:}

This is an area where there are no appropriate in vitro alternatives to in vivo models for excretion ( $\mathrm{Li}$ 2005). Hence excretion has not been studied in detail in an in vitro setting.

\section{E. Toxicity:}

There is no specific FDA guidance document for in vitro toxicity testing, therefore small customized safety panels are created for each drug and used for early screening for toxicity Different cell lines created from hepatocytes, cardiomyocytes and skeletal myocytes can be studied for organ-specific toxicity ( $\mathrm{Li}$ 2005). Enzyme based and receptor binding assays can be part of the testing panel too. Even though assays can vary, the most commonly measured endpoints are mentioned below.

* Membrane integrity (Li 2005): Usually, lactate dehyrogenase levels are measured to evaluate membrane integrity ( $\mathrm{Li} 2005)$ as they might be increased due to a damaged membrane ( $\mathrm{Ng}$ et al.). Hemolysis assays are used to screen for any degradation of red blood cells by the drug. An alternative endpoint is "cellular metabolite content" where bioluminiscence assays using luciferin can be employed to measure intracellular ATP which can be decreased if the cell is damaged or dead (Li 2005).

* Mitochondrial function ( $\mathrm{Li}$ 2005): The MTT assay is usually used to measure cytotoxicity; as normal cells will reduce MTT to purple crystals (Li 2005). Absorbance can be measured and correlated to cell number and cell death (Zhang et al.).

- Lysosomal functions (Li 2005): Neutral red dye is taken up by live cells through lysosomes but damaged cells will show decreased uptake (Li 2005).

* Mutagenic potential: The Ames test is used to screen for carcinogens by investigating the potential of the drug to reverse mutations. Bacteria containing a defective mutant gene which renders them unable to grow in media without histidine are plated with the drug (Sakai et al.). If this mutation is reversed, then these bacteria will be able to grow in the histidine-free media (Sakai et al.). This assay would be usually performed late in the lead optimization phase, unless there were reasons to believe that there might be mutagenic potential (e.g., for nucleoside analogs, probably incorporated earlier than normal).

* HERG assay: The hERG (human Ether a go-go Related Gene) is essential for creating potassium channels in the heart which in turn, are necessary for its proper functioning. Since abnormal heart rhythm or arrhythmias can be caused by various drugs due to blockages of these potassium channels, it is important to check whether they inhibit the current produced by these channels ('hERG Safety Assay'). Chinese Hamster Ovary (CHO) cells transfected with hERG are used. In a single well of a 384 well plate, such a cell is used to block the gap between two fluid-containing chambers. A pore forming 
agent is added to create a pore i.e. a channel in this cell. The test compound is then added to screen for inhibition. Electric current before and after addition of the compound is measured and translated to an $\mathrm{IC}_{50}$ value.

\section{In vivo ADME/T assays:}

\section{A. ADME}

Although most of the useful data for IND filing obtained from in vivo models relates to toxicity, the ADME analysis of these animals is useful in predicting the PK parameters for phase I clinical trials as well. These animal studies are generally conducted on two species, one rodent and one larger animal (usually rabbit, dog, pig or primate). These two points are used to extrapolate (or interpolate) to determine parameters for clinical administration. Absorption is easily determined by monitoring the concentration of the drug in the blood plasma after dosing and at regular time intervals thereafter. Distribution to different tissues can be observed by administering a radiolabeled agent and tracking this through the various systems and organs using body scans. Metabolite formation is determined using LC-MS to analyze blood samples. The use of an isotopic agent (such as a radiolabel) makes it easy to identify metabolites, as there will be dual peaks corresponding to the labeled and naturally abundant agents in the mass spectrum. Chemists then use this MS data to hypothesize how a drug might be metabolized in humans. Similarly, this same procedure can be used to determine excretion products, with these specimens coming from feces or urine instead of blood. In this fashion, the ADME data derived from animal studies allows biologists and medical doctors to estimate the appropriate does in humans. This also provides analytical chemists with an idea of what metabolites they might observe in the clinic.

\section{B. Toxicology}

Preclinical in vivo toxicology studies need to be conducted in two species of animals for both acute and chronic exposure. Rats and mice are the preferred first subjects used in vivo due to their body mass and extensive knowledge of these biological systems. Dogs, pigs and primates are the other preferred species for in vivo studies. Acute toxicity studies generally last about 2-4 weeks whereas chronic studies are anywhere between one month and one year in duration. Chronic studies need not be conducted in preparation for Phase I clinical trials. Furthermore, the length of these studies will depend up on the intended length of treatment in patients which varies between indications. The ICH recommends 6 month studies using rodents and 9 month studies when using other species for most chronic toxicology studies ('Duration of Chronic Toxicity Testing in Animals' 1998).

Toxic effects resulting from acute exposure is determined by monitoring drug levels and metabolites in the blood during exposure ('Note for Guidance on Toxicokinetics' 1994; Birendra, Afzal, Sochaki, et al. 2015; Birendra, Afzal, Wentland, et al. 2015) as well as necropsy of the test subjects when necessary. For these tests, only one sex may be used if there is no reason to believe there is a difference between genders or if the drug is only to be administered clinically to one gender ('Guidance on Genotoxicity Testing and Data Interpretation' 2008). If the test subject(s) die during the acute toxicology testing, the organs are harvested and examined by a certified veterinarian pathologist to determine details about injury to specific organs. If the test subjects do not display any toxicity as a result of the study, their lives may be spared and potentially used again for other toxicology studies. For genotoxicity, usually two experiments need to be conducted. First, bacterial mutation in vitro assays (Ames) are performed ('Guidance on Genotoxicity Testing and Data Interpretation' 2008), which are predictive of rodent carcinogenicity. Additional assays to assess genotoxicity include the DNA double-strand break assays (including "Comet"), the alkaline elution assay, DNA covalent-binding assays and unscheduled DNA synthesis assay from liver ('Testing of Carcinogenecity of Pharmaceuticals' 1997). This battery test approach is designed to reduce the risk of false negative results for compounds with genotoxic potential; however, a positive result in any assay for genotoxicity does not necessarily mean that the test compound poses a genotoxic or carcinogenic hazard to humans ('Guidance on Genotoxicity Testing and Data Interpretation' 2008). As such, this approach uses genotoxicity results to determine if carcinogenicity studies need to be conducted.

Since carcinogenicity studies are time consuming and resource intensive they are only being performed when human exposure warrants the need for information from life-time studies in animals in order to assess carcinogenic potential ('Need for Carcinogenecity Studies of Pharmaceuticals' 1995). Carcinogenicity studies are also conducted in two species by administering the drug via the same anticipated route of that to be used in humans. In the absence of clear evidence favoring one species, it is recommended that the rat be selected as one species ('Testing of Carcinogenecity of Pharmaceuticals' 1997). Most filings with the FDA conduct carcinogenicity studies for treatments which are expected to be administered routinely in excess of 3-month dosages in humans. 
Carcinogenicity testing is nearly universal for drugs administered 6-months or longer. Even if the anticipated therapeutic dosing does not meet or exceed these lengths, carcinogenicity studies are recommended for drugs that fall within certain categories of compounds known to be carcinogenic, when SARS suggests potential carcinogenicity or if pre-neoplastic lesions are observed in repeated dose studies. Experiments generally include noting both cellular changes and biochemical measurements ('Testing of Carcinogenecity of Pharmaceuticals' 1997). Cellular changes may include observations involving the morphological, histochemical or functional attributes of the relevant tissues. Biochemical measurements may include hormone levels, growth factors and enzyme activity. Unequivocally genotoxic compounds, in the absence of other data, are presumed to be transspecies carcinogens, thus implying a hazard to humans ('Need for Carcinogenecity Studies of Pharmaceuticals' 1995).

Reproductive toxicology is tricky as the PK parameters for pregnant or lactating animals will be different and is unlikely that this data will be available ('Note for Guidance on Toxicokinetics' 1994). For male reproductive toxicity, fertility is the focus of such studies. Given the complexity of these tests and the scope of this report, further discussion involving reproductive toxicology will be omitted.

\section{D Tissue Culture for ADME/T Studies}

\section{A. Need of 3D culture for Drug discovery}

In the pharmaceutical industry, late ailure of developmental candidates is a cause of concern. Such failure translates into loss of millions of dollars and several years of wasted time and efforts. This is primarily the reason why they prefer to identify "failure" drug molecule in early developmental stages. To accomplish this goal, researcher must develop novel and state of the art efficient assays and methodologies to better characterize these prospective drug molecules. Traditional state of art in vitro methodologies to characterize molecules in ADME/T processes have been $2 \mathrm{D}$ cell cultures. The 2D monolayer culture of hepatocyte and other cell type such as HEK 293T cells are relatively cheap and convenient to use for high throughput screening to identify hepatotoxicity and other ADME/T properties of drugs(Singh, Simpson, and Bennett 2015; Singh and Bennett 2009). In in vitro $\mathrm{ADME} / \mathrm{T}$ studies using hepatocyte monolayers on hard plastic or glass surface, it is assumed that the monolayer reflects the essential physiology of liver. In fact, liver-specific architecture, mechanical and biochemical characteristics and cell-cell communication are lost under simplified in vitro conditions. More than $50 \%$ of drugs tested in vitro in 2D hepatocytes fails to show their in vivo effects because of the lack of entire physiological system. For example, consider lack of idiosyncratic hepatotoxicity $(\mathrm{IH})$ in Invitro, a result of lack of immune-mediated reactions (Gomez-Lechon et al. 2010). An improved in vitro method of liver that could mimic in vivo liver functions and could predict drug pharmacokinetics and pharmacodynamics in an in vitro environment is highly desirable.

It is hypothesized that 3D cell culture that closely reflects in vivo environment will better predict to characterize pharmacokinetics and pharmacodynamics of different chemical entities. This early characterization of ADME/T properties will help drug researchers to exclude unpromising molecules early enough to save time and money.

\section{B. Advantages of 3D Tissue models over 2D models}

A reliable ex vivo information of physiological relevance can be offered by $3 \mathrm{D}$ cell cultures because 3D scaffolds reduce the gap between hepatocyte cell culture and resembling close to physiological liver. Liver consists on many types of cells including hepatocytes (main metabolic cells with in vivo value 60\%), endothelial cells (secrete cytokines with in vivo value $20 \%$ ), kupffer cells (macrophages, inflammatory response in vivo value $15 \%$ ), and stellate cells (fibroblasts, store fat and secrete ECM in vivo value 5\%) (Dash et al. 2009). These different cell types interact with neighboring cells and with ECM through biochemical and mechanical cues to establish a 3D communication network that maintains specificity and homeostasis of the tissue. Key events of cellular uptake of bio molecules and drugs, their metabolism, regulation of gene expression such as CYP and other metabolism related enzymes, signaling through ECM all together compiles into a complex network of information exchange to derive a process including drug metabolism into many intermediatory bioactive products as well as their later excretion.

\section{Hepatocyte 3D culture}

Primary hepatocytes within hydrogel of extracel are used for 3D culture, to evaluate hepatotoxicity in vitro (Prestwich 2008). These primary hepatocytes using collagen or matrigel(Knop et al. 1995; Bissell et al. 1987) 3D culture sandwich techniques retain their biochemistry and have enhanced longevity (Prestwich 2008). The sandwich model provides cell-cell interaction, cellextracellular matrix interaction, maintain polarity and morphology (Liu, LeCluyse, et al. 1999; Liu, 
Chism, et al. 1999; Marion, Leslie, and Brouwer 2007). These 3D hepatocytes maintain their metabolic competence but show some degree of reduction of phase I activity and gene expression (Mathijs et al. 2009). Later, an overlay sandwich culture has been developed that reduces shear stress and preserve mass transport consistency using laminar-flow perfusion(Ng et al. 2006). Another sandwich model is based on ultra-thin micro fabricated porous silicon nitride membrane. These models have improved mass transport features with decreased stress markers and enhanced liver like functions (Zhang et al. 2008; Bennett et al. 2014). Sandwich-cultured hepatocytes are limited to mechanistic studies of drug toxicity in association to transportation and partial identification of major intermediate metabolites(Meng 2010). This is because of gradual loss of metabolic enzyme CYP 2E1.

Spheroid Cultures: Another 3D version is spheroid cultures with microvilli projections expressing plasma proteins such as albumin and transferrin (Sakai et al. 1996; Tong et al. 1992). These spheroids have liver like histology but have limited transport of oxygen and other nutrition. These spheroids show higher sensitivity to drug hepatotoxicity than regular 2D cultures (Du et al. 2007), but the tightly associated spheroids showed different endpoints for drug hepatotoxicity as compared to $2 \mathrm{D}$. It was suggested that lack of compound penetration into these tight spheroids may limit their use in chronic in vitro toxicity assays (Meng 2010; Walker, Rhodes, and Westmoreland 2000).

Gel Entrapment Culture: is a semi-permeable hollow fiber cartridge with separate cells from the perfusion compartment and is a scaffold for hepatocyte entrapment (Tzanakakis et al. 2000). In gel entrapment culture medium circulation system provides nutrition and oxygen. Gel entrapped hepatocytes are more sensitive than 2D culture. expression (Shen et al. 2006; Meng et al. 2007). This model is sensitive to increased oxygen species production and mitochondrial dysfunction to account for hepatotoxicity induced by drug (Shen et al. 2009).

Hollow Fiber Bioreactors (HFB): HFB is an alternative to conventional cell culture methods and is being used for many PK studies. Cells grow on and around the large surface area provided by the network of hollow fibers. Hollow fiber cell and tissue culturing maximize surface area to minimize the volume of culture medium required. The porous hollow fibers form a 3 dimensional network of capillaries that continually delivers a supply of fresh media and nutrients to the cultured cells that remain isolated in the Extra- Capillary Space. The fiber membrane provides a selectively permeable barrier that permits passage of metabolic byproducts away from the cells while culturedependent nutrients permeate into the culture. When perfused with culture media, the hollow fibers allow oxygen and nutrients to be supplied to the cells while metabolic waste products are eliminated. The process increases the accumulation of the cell-secreted growth factors required for optimal growth. Due to the system's efficient delivery of media and removal of waste, the process uses less culture media than other methods. Some of the salient features of HFB are its support to many human cell types, promoting high cell density, supporting cell-cell interaction, low sheer, fluid mechanics imitating in vivo interstitial flow, controllable media flow \& exchange, and improved pharmacodynamics potential (Whitford and Cadwell 2009)

\section{Precision Cut Tissue Slices:}

Precision cut tissue slice (PCTS) is a process whereby cores of excised tissue, such as liver can be sliced into discs of uniform thickness. Both phase I and phase II metabolizing systems are intact (Heinonen et al. 1996) and inducible in PCTS (Lupp et al. 2002). Some advantages to this system include an intact organotypic architecture that is similar in composition to the original tissue. This allows evaluation of cell-to- cell interactions in vitro. Thus, PCTS is an excellent model for evaluating drugs of chemically-induced hepatobiliary toxicity (Amin et al. 2006). The system can be applied to problems that require the ability to discern toxicity, including target organ toxicity, and species-specific toxicity (Vickers and Fisher 2005; Parrish, Gandolfi, and Brendel 1995; Lerche-Langrand and Toutain 2000; Rodrigues et al. 1994).

\section{SUMMARY}

Development of small molecules is full of challenges but pioneering ADMET investigations on new approaches could advance our knowledge in drug discovery. A better understanding of ADME processes, by pharmacologists in early discovery through late development, could be crucial to enhance success rate and reduce the cost of drug molecules. In particular, predicting toxic effects of preclinical molecules have been the major challenge in early stage of drug cycle. Though, predictive in silico or rule-based ADME tools could boost the discovery and reduce time as well as expense in early identification stages, yet better pre-clinical assays are in need to be developed. Recently, scientists have shown a lot of interest in $3 \mathrm{D}$ cell culture models as compared to 2D cell cultures because they could better replicate in vivo conditions.

Conflict of Interest: None 


\section{REFERENCES}

1. Adams, C.P., and V.V. Brantner. 2010. 'Spending on new drug development1', Health Econ, 19: 13041.

2. Amin, K.,C. Ip, B. Sato, T.Le, C.E. Green, C. A. Tyson, and H. P. Behrsing. 2006. 'Characterization of ANIT-induced toxicity using precision-cut rat and dog liver slices cultured in a dynamic organ roller system', Toxicol Pathol, 34: 776-84.

3. Bennett, R. G., D. G. Heimann, S. Singh, R. L. Simpson, and D. J. Tuma. 2014. 'Relaxin decreases the severity of established hepatic fibrosis in mice', Liver Int, 34: 416-26.

4. Birendra, K. C., M. Z. Afzal, A. Sochaki, K. A. Wentland, R. Chang, S. Singh, and T. O'Rourke. 2015. 'Tumor molecular profiling in the treatment of refractory cancers', J Exp Ther Oncol, 11: 27-32.

5. Birendra, K. C., M. Z. Afzal, K. A. Wentland, H. Hashmi, S. Singh, E. Ivan, and N. Lakhani. 2015. 'Spontaneous Regression of Refractory Diffuse Large B-Cell Lymphoma with Improvement in Immune Status with ART in a Patient with HIV: A Case Report and Literature Review', Am J Case Rep, 16: 347-52.

6. Bissell, D. M., D. M. Arenson, J. J. Maher, and F. J. Roll. 1987. 'Support of cultured hepatocytes by a laminin-rich gel. Evidence for a functionally significant subendothelial matrix in normal rat liver', $J$ Clin Invest, 79: 801-12.

7. Brown, Nicholas F., Maja Stefanovic-Racic, Ian J. Sipula, and German Perdomo. 2007. 'The mammalian target of rapamycin regulates lipid metabolism in primary cultures of rat hepatocytes', Metabolism, 56: 1500-07.

8. Dash, A., W. Inman, K. Hoffmaster, S. Sevidal, J. Kelly, R. S. Obach, L. G. Griffith, and S. R. Tannenbaum. 2009. 'Liver tissue engineering in the evaluation of drug safety', Expert Opin Drug Metab Toxicol, 5: 1159-74.

9. DiMasi, J. A., R. W. Hansen, and H. G. Grabowski. 2003. 'The price of innovation: new estimates of drug development costs', J Health Econ, 22: 151- 85.

10. Du, Y., R. Han, S. Ng, J. Ni, W. Sun, T. Wohland, S.H. Ong, L. Kuleshova, and H. Yu. 2007. 'Identification and characterization of a novel prespheroid 3-dimensional hepatocyte monolayer on galactosylated substratum', Tissue Eng, 13: 1455-68.'Duration of Chronic Toxicity Testing in Animals'.1998.

11. http://www.ich.org/products/guidelines/safety/safety-single/article/duration-of-chronic-toxicity-testingin-animals-rodent-and-non-rodent-toxicity- testing.html.

12. Gomez-Lechon, M. J., A. Lahoz, L. Gombau, J. V. Castell, and M. T. Donato. 2010. 'In vitro evaluation of potential hepatotoxicity induced by drugs', Curr Pharm Des, 16: 1963-77.

13. 'Guidance on Genotoxicity Testing and Data Interpretation'. 2008.

14. http://www.ich.org/products/guidelines/safety/safet y-single/article/guidance-on-genotoxicity-testingand-data-interpretation-for-pharmaceuticals- intended-for-human-use.html.

15. Guttendorf, Robert J. 'The Emerging Role of A.D.M.E. in Optimizing Drug Discovery and Design'. http://www.netsci.org/Science/Special/feature06.ht ml Heinonen, J. T., J. S. Sidhu, M. T. Reilly, F. M. Farin,

16. J. Omiecinski, D. L. Eaton, and T. J. Kavanagh. 1996. 'Assessment of regional cytochrome P450 activities in rat liver slices using resorufin substrates and fluorescence confocal laser cytometry', Environ Health Perspect, 104: 536-43.

17. 'Herg Safety Assay'. http://www.cyprotex.com/toxicology/early- toxicity/hergsafety/.

18. Hewitt, N. J., M. J. Lechon, J. B. Houston, D. Hallifax, H. S. Brown, P. Maurel, J. G. Kenna, L. Gustavsson, C. Lohmann, C. Skonberg, A. Guillouzo, G. Tuschl, A. P. Li, E. LeCluyse, G. M. Groothuis, and J. G. Hengstler. 2007. 'Primary hepatocytes: current understanding of the regulation of metabolic enzymes and transporter proteins, and pharmaceutical practice for the use of hepatocytes in metabolism, enzyme induction, transporter, clearance, and hepatotoxicity studies', Drug Metab Rev, 39: 159-234.

19. Ito, K., H. S. Brown, and J. B. Houston. 2004. 'Database analyses for the prediction of in vivo drugdrug interactions from in vitro data', Br J Clin Pharmacol, 57: 473-86.

20. Ito, Kiyomi, David Hallifax, R. Scott Obach, and J. Brian Houston. 2005. 'Impact of Parallel Pathways of Drug Elimination and Multiple Cytochrome P450 Involvement on Drug-Drug Interctions: CYP2D6 Paradigm', Drug Metabolism and Disposition, 33: 837-44.

21. Kaminski, N. E., and W. D. Stevens. 1992. 'The role of metabolism in carbon tetrachloride-mediated immunosuppression. In vitro studies', Toxicology, 75: 175-88.

22. Kletter, D., S. Singh, M. Bern, and B. B. Haab. 2013. 'Global comparisons of lectin-glycan interactions using a database of analyzed glycan array data', Mol Cell Proteomics, 12: 1026-35. 
23. Knop, E., A. Bader, K. Boker, R. Pichlmayr, and K. F. Sewing. 1995. 'Ultrastructural and functional differentiation of hepatocytes under long-term culture conditions', Anat Rec, 242: 337-49.

24. Kola, Ismail, and John Landis. 2004. 'Can the pharmaceutical industry reduce attrition rates?', Nat Rev Drug Discov, 3: 711-16.

25. Lerche-Langrand, C., and H. J. Toutain. 2000. 'Precision-cut liver slices: characteristics and use for in vitro pharmaco-toxicology', Toxicology, 153: 221-53.

26. Li, Albert P. 2005. 'Preclinical in vitro screening assays for drug like properties', Drug Discovery today-Technologies, 2.Li, Xia, Zhi-Chao Qu, and James M. May. 2001. 'GSH Is Required to Recycle Ascorbic Acid in Cultured Liver Cell Lines', Antioxidants \& Redox Signaling, 3: 1089-97.

27. Liu, X., J. P. Chism, E. L. LeCluyse, K. R. Brouwer, and K. L. Brouwer. 1999. 'Correlation of biliary excretion in sandwich-cultured rat hepatocytes and in vivo in rats', Drug Metab Dispos, 27: 637-44.

28. Liu, X., E. L. LeCluyse, K. R. Brouwer, L. S. Gan, J.

29. J. Lemasters, B. Stieger, P. J. Meier, and K. L. Brouwer. 1999. 'Biliary excretion in primary rat hepatocytes cultured in a collagen-sandwich configuration', Am J Physiol, 277: G12-21.

30. Lupp, A., R. Glockner, M. Danz, and D. Muller. 2002. 'Cryopreserved precision-cut rat liver slices: morphology and cytochrome P450 isoforms expression after prolonged incubation', Toxicol In Vitro, 16: 749-58.

31. Maggs, J. L., L. P. Bishop, K. T. Batty, C. C. Dodd, K. F. Ilett, P. M. O'Neill, G. Edwards, and B. Kevin Park. 2004. 'Hepatocellular bioactivation and cytotoxicity of the synthetic endoperoxide antimalarial arteflene', Chem Biol Interact, 147: 173-84.

32. Marion, T. L., E. M. Leslie, and K. L. Brouwer. 2007. 'Use of sandwich-cultured hepatocytes to evaluate impaired bile acid transport as a mechanism of drug-induced hepatotoxicity', Mol Pharm, 4: 911-8.

33. Mathijs, K., A. S. Kienhuis, K. J. Brauers, D. G. Jennen, A. Lahoz, J. C. Kleinjans, and J. H. van Delft. 2009. 'Assessing the metabolic competence of sandwich-cultured mouse primary hepatocytes', Drug Metab Dispos, 37: 1305-11.

34. McCarter, C., D. Kletter, H. Tang, K. Partyka, Y. Ma, S. Singh, J. Yadav, M. Bern, and B. B. Haab. 2013. 'Prediction of glycan motifs using quantitative analysis of multi-lectin binding: Motifs on MUC1 produced by cultured pancreatic cancer cells', Proteomics Clin Appl, 7: 632-41.

35. McKim, J. M., Jr. 2010. 'Building a tiered approach to in vitro predictive toxicity screening: a focus on assays with in vivo relevance', Combinatorial chemistry \& high throughput screening, 13: 188- 206.

36. Meng, Q. 2010. 'Three-dimensional culture of hepatocytes for prediction of drug-induced hepatotoxicity', Expert Opin Drug Metab Toxicol, 6: 733-46.

37. Meng, Q., J. Ru, G. Zhang, C. Shen, S. Schmitmeier, and A. Bader. 2007. 'Re-evaluation of tacrine hepatotoxicity using gel entrapped hepatocytes', Toxicol Lett, 168: 140-7.

38. Naritomi, Yoichi, Shigeyuki Terashita, Akira Kagayama, and Yuichi Sugiyama. 2003. 'Utility of

39. Hepatocytes in Predicting Drug Metabolism: Comparison of Hepatic Intrinsic Clearance in Rats and Humans in vivo and in Vitro', Drug Metabolism and Disposition, 31: 580-88.

40. 'Need for Carcinogenecity Studies of Pharmaceuticals'. 1995.

41. http://www.ich.org/products/guidelines/safety/safet $\quad \mathrm{y}$-single/article/need-for-carcinogenicity-studiesof-pharmaceuticals.html.

42. Ng, S., R. Han, S. Chang, J. Ni, W. Hunziker, A. B. Goryachev, S. H. Ong, and H. Yu. 2006. 'Improved hepatocyte excretory function by immediate presentation of polarity cues', Tissue Eng, 12: 2181-91.

43. 'Note for Guidance on Toxicokinetics'. 1994. http://www.ich.org/products/guidelines/safety/safet y-single/article/note-for-guidance-ontoxicokinetics-the-assessment-of-systemic- exposure-in-toxicity-studies.html.

44. Parrish, A. R., A. J. Gandolfi, and K. Brendel. 1995. 'Precision-cut tissue slices: applications in pharmacology and toxicology', Life Sci, 57: 1887- 901.

45. 'Preclinical Safety Evaluation of Biotechnology- Derived Pharmaceuticals'. 2011.

46. http://www.ich.org/products/guidelines/safety/safet $\quad$-single/article/preclinical-safety-evaluation-ofbiotechnology-derived-pharmaceuticals.html.

47. Prestwich, G. D. 2008. 'Evaluating drug efficacy and toxicology in three dimensions: using synthetic extracellular matrices in drug discovery', Acc Chem Res, 41: 139-48.

48. Rodrigues, A. D., J. L. Ferrero, M. T. Amann, G. A. Rotert, S. P. Cepa, B. W. Surber, J. M. Machinist,

49. N. R. Tich, J. P. Sullivan, D. S. Garvey, and et al. 1994. 'The in vitro hepatic metabolism of ABT- 418, a cholinergic channel activator, in rats, dogs, cynomolgus monkeys, and humans', Drug Metab Dispos, 22: 788-98.

50. Sakai, Y., K. Naruse, I. Nagashima, T. Muto, and M. Suzuki. 1996. 'Large-scale preparation and function of porcine hepatocyte spheroids', Int J Artif Organs, 19: 294-301. 
51. Shen, C., X. Cheng, D. Li, and Q. Meng. 2009. 'Investigation of rifampicin-induced hepatotoxicity in rat hepatocytes maintained in gel entrapment culture', Cell Biol Toxicol, 25: 265-74.

52. Shen, C., H. Zhang, G. Zhang, and Q. Meng. 2006. 'Isoniazid-induced hepatotoxicity in rat hepatocytes of gel entrapment culture', Toxicol Lett, 167: 66-74.

53. Singh, S., and R. G. Bennett. 2009. 'Relaxin family peptide receptor 1 activation stimulates peroxisome proliferator-activated receptor gamma', Ann N Y Acad Sci, 1160: 112-6.

54. Singh, S., R. L. Simpson, and R. G. Bennett. 2015. 'Relaxin activates peroxisome proliferator-activated receptor gamma (PPARgamma) through a pathway involving PPARgamma coactivator alpha (PGC1alpha)', J Biol Chem, 290: 950-9.

55. Steinmetz, K. L., and E. G. Spack. 2009. 'The basics of preclinical drug development for neurodegenerative disease indications', BMC Neurol, 9 Suppl 1: S2.

56. 'Testing of Carcinogenecity of Pharmaceuticals'. 1997.

http://www.ich.org/products/guidelines/safety/safety-single/article/testing-for-carcinogenicity-ofpharmaceuticals.html.

57. Tong, J. Z., P. De Lagausie, V. Furlan, T. Cresteil, O. Bernard, and F. Alvarez. 1992. 'Long-term culture of adult rat hepatocyte spheroids', Exp Cell Res, 200: 326-32.

58. Toutain, H. J., V. Moronvalle-Halley, J. P. Sarsat, C. Chelin, D. Hoet, and D. Leroy. 1998. 'Morphological and functional integrity of precision-cut rat liver slices in rotating organ culture and multiwell plate culture: effects of oxygen tension', Cell Biol Toxicol, 14: 175-90.

59. Tzanakakis, E. S., D. J. Hess, T. D. Sielaff, and W. S. Hu. 2000. 'Extracorporeal tissue engineered liver- assist devices', Annu Rev Biomed Eng, 2: 607-32.

60. Vickers, A. E., and R. L. Fisher. 2005. 'Precision-cut organ slices to investigate target organ injury', Expert Opin Drug Metab Toxicol, 1: 687-99.

61. Walker, T. M., P. C. Rhodes, and C. Westmoreland. 2000. 'The differential cytotoxicity of methotrexate in rat hepatocyte monolayer and spheroid cultures', Toxicol In Vitro, 14: 475-85.

62. Whitford, W. G., and J. J. S. Cadwell. 2009. 'Interest In Hollow-Fiber Perfusion Bioreactors is Growing'. http://www.fibercellsystems.com/documents/Hollo wFiberPerfusion.pdf.

63. Wienkers, Larry C., and Timothy G. Heath. 2005. 'Predicting in vivo drug interactions from in vitro drug discovery data', Nat Rev Drug Discov, 4: 825- 33.

64. Wilkening, S., F. Stahl, and A. Bader. 2003. 'Comparison of primary human hepatocytes and hepatoma cell line Hepg 2 with regard to their biotransformation properties', Drug Metab Dispos, 31: 1035-42.

65. Zhang, S., L. Xia, C. H. Kang, G. Xiao, S. M. Ong, Y. Toh, H. L. Leo, D. van Noort, S. H. Kan, H. H. Tang, and H. Yu. 2008. 'Microfabricated silicon nitride membranes for hepatocyte sandwich culture', Biomaterials, 29: 3993-4002. 